\title{
On Detection of Observation Faults in the Observation and Position Domains for Positioning of Intelligent Transport Systems
}

\author{
Ahmed El-Mowafy \\ School of Earth and Planetary Sciences, \\ Curtin University, Australia \\ GPO Box U 1987, Perth WA 6845, Australia \\ Tel | +61892663403 \\ Fax $\mid+61892662703$ \\ Email|a.el-mowafy@curtin.edu.au
}

\begin{abstract}
Intelligent Transportation Systems (ITS) depend on Global Navigation Satellite systems (GNSS) as a major positioning sensor, where the sensor should be able to detect and exclude faulty observations to support its reliability. In this article two fault detection and Exclusion (FDE) approaches are discussed. The first is its application in the observation domain using Chi-square test in Kalman filter processing. The second approach discusses FDE testing in the positioning domain using the solution separation (SS) method, where new FDE forms are presented that are tailored for ITS. In the first form the test is parameterized along the direction of motion of the vehicle and in the cross direction, which are relevant to applications that require lane identification and collision alert. A combined test is next established. Another form of the test is presented considering the maximum possible positioning error, and finally a direction-independent test. A new test that can be implemented in the urban environment is presented, which takes into account multipath effects that could disrupt the zero-mean normal-distribution assumption of the positioning errors. Additionally, a test is presented to check that the position error resulting from the remaining measurements lies within acceptable limits. The proposed methods are demonstrated through a kinematic test run in various environments that may be experienced in ITS.
\end{abstract}

\section{KEYWORDS}

GNSS, Positioning, Fault detection and Exclusion, Intelligent Transport systems.

\section{INTRODUCTION}

Intelligent Transport systems (ITS) require reliable vehicle positioning in real time. The Global Navigation Satellite Systems (GNSS) are widely used for this purpose and to achieve reliable vehicle localization, its positioning integrity should be monitored. Such integrity monitoring for transport applications has been addressed, for instance in Margaria and Falletti (2014), Sanat et al., (2006), and Zhu et al. (2018), where the focus was primarily on the use of single-frequency receivers. However, ITS applications require precise positioning at the sub-m accuracy, e.g. for lane identification in tolling, pay-as-you-drive insurance and priority traffic signal control systems, and dm accuracy for collision alert. Therefore, the use of dual-frequency and multi-constellation observations is needed.

In the harsh urban conditions, GNSS outage frequently appears due to insufficient number of observations. To deliver continuous positioning solutions, GNSS is typically supported by other navigation positioning sensors such as the Inertial Measurement Unit (IMU). In vehicular navigation, and due to cost limitations, the low-cost MicroElectro-Mechanical System (MEMS) sensors are often used (Aggarwal et al. 2010). In addition, the odometer and LiDAR sensors can be integrated with GNSS and IMU to improve the positioning performance. However, in this article we will restrict our focus to GNSS only.

In integrity monitoring (IM), two tasks are performed. In the first task, the system is monitored to detect and exclude faults in the observations or the system; this process is known as fault detection and exclusion (FDE). The second task is to inform the user (or more precisely the computer of the vehicle) if the system cannot meet pre-set 
requirements (El-Mowafy and Kubo 2017). FDE usually relies on statistical hypothesis testing by applying a consistency check among all possible sets of observations that can provide a solution. Hence, we can only detect faults when positioning can be available from at least one set of error-free observations, and thus redundant observations are needed. FDE has been proposed in several forms, some examples are the single-observation data snooping (Baarda 1968), the multi-observation detection-identification-adaptation (DIA) method (Teunissen 2006), Receiver Autonomous Integrity Monitoring (RAIM) (Parkinson and Axelrad 1987; Brown 1992), RAIM for multiple outliers (Knight et al., 2010), and Advanced RAIM (ARAIM) (Blanch et al. 2015).

Two approaches are usually used in FDE, the first is applied in the observation domain and the second is performed in the position domain. Assuming a normal distribution of the observation errors in the fault-free mode, one may test the validity of this hypothesis in the observation domain by checking that the sum-of-squared-residuals of the observations have a central Chi-square distribution (Sturza 1988; Walter and Enge 1995). The test checks the working hypothesis against specified alternative hypotheses, e.g. the presence of single or multiple satellite faults (Powe and Owen 1997). Depending on the parameterization of the underlying model, many implementations of the test-statistics exist (Teunissen 2000; Blanch et al. 2015; Imparato et al. 2018; Jöerger and Pervan 2016). Since in the least squares process or Kalman filtering a specific large error might be smoothed out through its neighbouring observations; methods such as 'Observation Subset Testing' were developed (Kuang, 1996; Kuusniemi, 2005), where consistency tests are computed for all the possible subsets by excluding suspected observations. When multiple observations are excluded the position model becomes weak; therefore, the Forward-Backward FDE method was introduced such that the iterated reliability checking includes a reconsideration of earlier rejected observations to ensure that the order of the excluded measurements does not cause an unnecessary exclusion (Wieser, 2001). Another method, known as the Danish method, iteratively reweights the observations if the magnitude of a residual is outside a defined range, in consequence excluding bad observations by reducing their weights, until the solution converges (Krarup, 1980; Leick, 2004). Although this method may computationally be efficient when dealing with multiple faults compared with other exclusion methods, the drawback of the Danish method is that it is purely heuristic with no rigorous statistical theory (Leick, 2004).

In the position domain, fault detection can be performed using the solution separation method. In this method, a position error bound is created for each possible fault mode by computing a position solution unaffected by the fault, computing an error bound around this solution and accounting for the difference between the all-observations position solution and the fault tolerant position (Blanch et al. 2015). The method has been applied within the framework of ARAIM, which is an evolution of classical RAIM, in which multi-frequency multi-constellation observations are considered, with the possibility of detection multiple faults (Walter et al. 2013; El-Mowafy and Yang 2016; El-Mowafy 2017). Sine in ITS multi-frequency multi-constellation observations are employed to achieve precise positioning, the use of ARAIM is considered, which is typically performed in the position domain. Therefore, the two approaches applying the FDE in the observation and position domains will be addressed, but more focus will be given to the latter.

The applications of ITS vary widely, and positioning can be presented in different ways according to the application at hand. For instance, the position of a vehicle is presented on the navigation maps using the Easting and Northing coordinates. On the other hand, in the liability-critical applications, such as tolling, the cross-track position is important for lane identification. In safety-critical applications, such as collision avoidance, the position is better expressed in terms of the vehicle direction of motion, i.e. in the along-track and cross-track directions. Another case when the direction of the vehicle is changing rapidly, e.g. during turns. The use of one FDE model for the different positioning presentations in all these scenarios is not optimal, therefore, the FDE in this contribution presents new testing models for these possible scenarios. Moreover, the vehicle can operate in open sky or in urban areas. FDE tests typically assume zero-mean Gaussian distribution of the observation and position errors, which is acceptable in open sky environments. However, this assumption may be invalid in the urban environment due to the presence of significant multipath, therefore, a new test is presented specifically for this environment, taking into consideration multipath effects. The switch between these testing models can be easily performed by the computer of the ITS vehicle according to the vehicle status and application. In addition, a test defined as the Final Integrity Test (FIT) is presented that quantifies the impact of the remaining measurement and system errors after the FDE process on the final position (El-Mowafy and Imparato 2018), and whether the resulting position error lies within an acceptable range, which is set according to the ITS application considered.

The next sections present the FDE parameterization and tests when being applied firstly in the observation domain and secondly in the position domain with a focus on ITS applications. The presented methods can be applied in real time. Next, these methods are applied in a kinematic test that was conducted under varying work environments including suburban, urban and near to tree canopies. Their results are then analysed and compared. 


\section{THE NULL AND ALTERNATIVE HYPOTHESES}

Restricting our interest to positioning using GNSS only, the fault-free observation equation in a Kalman filtering $(\mathrm{KF})$ setting at time $t$ in a linearized model can be expressed as:

$$
y_{t}=G_{t} x_{t / t-1}+v_{t}
$$

where $y_{t}$ is the measurement vector, computed as the difference between the observations and their estimated values. The latter is computed from the approximate position of the user along the route and satellite positions. $x$ is the unknown vector of states, which mainly includes the difference between the final and approximate vehicle positions (in addition to other unknowns such as the receiver clock offset, etc.), and $x_{t / t-1}$ is its time update. $G_{t}$ is the geometry (design) matrix. $v_{t}$ is the time-updated (predicted) observation residuals, also known as the vector of innovations, which are uncorrelated in time. The fault-free (null) hypothesis is expressed as $H_{0}: E\left\{y_{t}\right\}=G_{t} \hat{x}_{t}$ with $\mathrm{D}\left\{y_{t}\right\}=Q_{y}$, representing the variance-covariance $(\mathrm{VC})$ matrix of the observations, where $\mathrm{E}\{\}$ and $\mathrm{D}\{\}$ denote the expectation and dispersion operators.

In the presence of suspected faults, or large errors, denoted here as $\nabla_{t}$, the observation model can be expressed as (Teunissen and Kleusberg 1998):

$$
y_{t}=G_{t} x_{t / t-1}+C_{t} \nabla_{t}+v_{t}
$$

which gives the alternative hypothesis $H_{a}: \mathrm{E}\left\{y_{t}\right\}=G_{t} \hat{x}_{t}+C_{t} \nabla_{t}$, where $C_{t}$ is a matrix that describes all possibilities of observations suspected to be faulty. This may include a blunder in a code observation or a slip in a phase observation. For $m$ observations, we can detect up to $q_{m}$ number of faults such that $1 \leq q_{m} \leq d f$, where $d f$ is the degrees of freedom. $C_{t}$ will then be $m \times q_{m}$ matrix, where each of its columns has a one corresponding to the observation assumed to be affected by a fault and zeros elsewhere. For Example, when examining the possibility of a fault in the first observation, $C_{t}=\left[\begin{array}{llll}1 & 0 & 0 & \ldots\end{array}\right]^{T}$, and when examining possible faults in the first and second observations $C_{t}=\left[\begin{array}{cccc}1 & 0 & 0 & \cdots \\ 0 & 1 & 0 & \cdots\end{array}\right]^{T}$.

In ITS, Satellite Based Augmentation Systems (SBAS) can be used for low accuracy applications (e.g. tolling) whereas real-time kinematic (RTK) or Network RTK would be needed for applications that require sub-m accuracy. With such accuracy, the use of float carrier-phase ambiguities will then be acceptable, thus, alleviating the need and risk of fixing the ambiguities. In the former method, the FDE will be concerned with code outliers whereas in RTK, and due to the correlation between phase and code errors, cycle slips of phase observations are first detected and repaired and next code outliers are excluded (El-Mowafy 2014). Several methods are presented for cycle slip detection, for instance by using the time difference of the between-phase observations from two frequencies or by monitoring the rate of change of the ionosphere delay.

\section{FDE IN THE OBSERVATIONS DOMAIN}

In the above model, one would be interested to know the value of the error vector $\nabla_{t}$. Its best estimator $\left(\hat{\nabla}_{t}\right)$ can be determined from (Teunissen 2006):

$$
\widehat{\nabla}_{t}=\left(C_{v_{t}}^{T} Q_{v_{t}}^{-1} C_{v_{t}}\right)^{-1} C_{v_{t}}^{T} Q_{v_{t}}^{-1} \hat{v}_{t}
$$

and its VC matrix $Q_{\widehat{\nabla}_{t}}$ is expressed as $Q_{\widehat{v}_{t}}=\left(C_{v_{t}}^{T} Q_{v_{t}}^{-1} C_{v_{t}}\right)^{-1}$ where the hat refers to estimated variables, and $Q_{\hat{v}}$ is the VC matrix of $\hat{v}$ determined as $Q_{\hat{v}}=Q_{\mathrm{y}}+\left[G_{t} P_{t, t-1} G_{t}^{T}\right]$, where $P_{t, t-1}$ is the VC matrix of the predicted states.

In this modelling scheme, the detection test statistic in FDE can be expressed as $T_{q}=\left(\hat{\nabla}^{T} Q_{\hat{\nabla}}^{-1} \hat{\nabla}\right)$. Under the assumption that the observation errors are zero-mean with a Gaussian distribution in the fault-free mode, $\widehat{\nabla}_{t}$ can also be assumed normally distributed and hence the statistic will have a Chi-square $\chi^{2}(d f, 0)$ distribution (the test will thus be defined as the Chi-square test). For detection of faults, one may suspect any observation, i.e. by setting $C_{v_{t}}$ to a unit matrix. Thus, the test statistic $T_{q}=\left(\hat{\nabla}^{T} Q_{\hat{\nabla}}^{-1} \hat{\nabla}\right)$ will reduce to $T_{q}=\left(\hat{v}^{T} Q_{\hat{v}}^{-1} \hat{v}\right)$, such that (Teunissen and Kleusberg 1998):

$$
H_{o}: T_{q}=\left(\hat{v}^{T} Q_{\hat{v}}^{-1} \hat{v}\right) \sim \chi_{\alpha}^{2}(d f, 0) ; \quad H_{a}: T_{q}=\left(\hat{v}^{T} Q_{\hat{v}}^{-1} \hat{v}\right) \sim \chi_{\alpha}^{2}(d f, \lambda)
$$

and we suspect the presence of a fault when 


$$
H_{o}: T_{q}=\left(\hat{v}^{T} Q_{\hat{v}}^{-1} \hat{v}\right) \geq \chi_{\alpha}^{2}(d f, 0) \quad \text { or equivalently if } \quad T_{q}=\frac{\left(\hat{v}^{T} Q_{\hat{v}}^{-1} \hat{v}\right)}{d f} \geq \mathrm{F}_{\alpha}(d f, \infty, 0)
$$

where $\lambda$ is the non-centrality parameter, and $F_{\alpha}$ is F-distribution threshold. In the 2D space, the allowable region in (4) represents an ellipse. The significance level $(\alpha)$ is set as the allowable false alarm rate and hence is aligned to the continuity requirement, which can be selected as $99 \%$ in ITS applications, giving a probability of false alarm $\alpha=1 \%$. This can be explained as follows. For $n$ number of satellites, there would be a total of $N=2^{n}-1$ possibilities of faults in single or combined observations. The probability of discontinuity $\left(\mathrm{P}_{\text {discont }}\right)$ will be:

$$
\mathrm{P}_{\text {discont }}=\alpha+\sum_{\mathrm{i}=1}^{\mathrm{N}} \mathrm{P}_{\mathrm{D}}\left(\mathrm{H}_{\mathrm{i}}\right) \mathrm{P}_{\mathrm{H}_{\mathrm{i}}}
$$

where $\alpha$ is the probability of false alarm, $\mathrm{P}_{\mathrm{D}}\left(\mathrm{H}_{\mathrm{i}}\right)$ is the probability of detection of faults in mode $\mathrm{i}$, which is less than 1 , and $\mathrm{P}_{\mathrm{H}_{\mathrm{i}}}$ is the prior probability of occurrence of this fault (e.g. $10^{-5} / \mathrm{h}$ for a single GPS satellite fault according to ICD-GPS-200J). The second term on the right hand side of Eq.(5) is thus too small, such that one can take $\mathrm{P}_{\text {discont }} \approx \alpha$.

When faults are detected, one needs to identify and exclude the observation(s) corresponding to the suspected faults, trying to identify among the multiple alternative hypotheses the most likely ones. The case of identifying a combination of possible faulty observations can also be performed through setting the suspected combinations in $C_{v_{t}}$ as explained in the previous section. Thus, the examined combination is suspected when the test statistic exceeds the threshold value, i.e. when:

$$
T_{q}=\left(\hat{\nabla}^{T} Q_{\widehat{\nabla}}^{-1} \hat{\nabla}\right) \geq \chi_{\alpha^{\prime}}^{2}(d f, 0) \quad \text { or equivalently } \quad T_{q}=\frac{\left(\widehat{\nabla}^{T} Q_{\bar{\nabla}}^{-1} \widehat{\nabla}\right)}{d f} \geq \mathrm{F}_{\alpha^{\prime}}(d f, \infty, 0)
$$

where $\alpha^{\prime}$ is the significance level in the identification test. For the case of identifying a single faulty observation e.g. for observation $j$, the test statistic reduces to $T_{q=1}=\frac{\widehat{\nabla}_{j}{ }^{2}}{\sigma_{\widehat{\nabla}_{j}}{ }^{2}}$, giving the normalised error $w_{j}=\frac{\widehat{\nabla}_{j}}{\sigma_{\widehat{\nabla}_{j}}}$, known as the $w$ statistic, where $\sigma_{\widehat{\nabla}_{j}}$ is the standard deviation of $\hat{\nabla}_{j}$ computed from the VC matrix $Q_{\widehat{\nabla}}$. We suspect a fault in observation $j$ in the fault mode $i$ when:

$$
w_{j}>N_{\frac{\alpha^{\prime}}{2 m}}(0,1) \text { and } w_{j} \geq w_{k} \quad \text { for } k=1 \text { to } m
$$

The threshold $N_{\frac{\alpha^{\prime}}{2 m}}(0,1)$ is the inverse of the complement of the standard normal distribution for $\frac{\alpha^{\prime}}{2 m}$, where we assume $\frac{\alpha^{\prime}}{2}$ is equally distributed among the $m$ observations. The $w$ values are ordered, and the observation with the largest $w_{j}$ value is excluded first.

However, the distribution of this significance level among different possible hypotheses is a complex issue, in particular for the cases of testing the possibility of the presence of more than one fault simultaneously. The simplest approach one can follow is to assume equal distribution of the total significance level among the alternative hypothesis as for the case of considering one single fault at a time. Let us restrict our focus to this case, and to determine $\alpha^{\prime}$ we use Baarda's B method (Baarda 1968), which assumes same probability for a type II error (i.e. failure to reject a false null hypothesis) in both the detection (using $\alpha$ ) and identification (using $\alpha^{\prime}$ ) tests. The method can be summarized as follows. We set the non-centrality parameters to be equal for the two cases such that:

$$
\lambda_{1}(\alpha, q=d f, \beta)=\lambda_{2}\left(\alpha^{\prime}, q=1, \beta\right)
$$

where $\lambda_{1}$ and $\lambda_{2}$ refer to the non-centrality parameter for the detection and identification tests, respectively. In ITS, and depending on the application at hand, we can select for example $\alpha=1 \%$ as mentioned earlier and a probability of miss-detection $\beta=10^{-5}$. Since in the B method $\alpha$ increases with the increase of redundancy, we start by considering $q=d f$ to limit our maximum $\alpha$ to the significance level $1 \%$. We first compute the left-hand side $\lambda_{1}$ from $\alpha, \beta$ and $d f$. One way to compute the non-centrality parameter is to consider the threshold value, denoted as $K$, by first computing $K \approx \chi_{\alpha}^{2}(d f, 0)$. Next, compute $\lambda_{1}$ by taking $\psi^{-1}(1-\beta)=k$ where $\psi^{-1}()$ is the inverse of the complement of $\chi_{\alpha}^{2}\left(d f, \lambda_{1}\right)$. Let us set equal values for the non-centrality parameters i.e. $\lambda_{2}=\lambda_{1}$, and the same for $\beta$. Then, in a backward way, take $q=1$, and iteratively solve for $\alpha^{\prime}$ from $\chi_{\alpha^{\prime}}^{2}\left(1, \lambda_{2}\right)=\psi^{-1}(1-\beta)$. This 
approach indicates that both the detection and identification tests will have the same minimum bias that can be detected with the chosen significance level, known as the minimal detectable bias (MDB) (De Bakker et al., 2009), i.e. the same reliability. Thus if the null hypothesis is accepted in the detection step, no further testing is needed (Teunissen 2006).

Before exclusion of any observation, a separability check can be applied by computing the correlation between faults to avoid masking of one fault by another. For example, for the observations $k$ and $j$, the correlation coefficient between their corresponding errors denoted as $\xi_{\widehat{\nabla}_{k}, \widehat{\nabla}_{j}}$ reads (El-Mowafy 2015):

$$
\xi_{\widehat{\nabla}_{k}, \widehat{\nabla}_{j}}=\frac{c_{v_{k}}^{T} Q_{v}^{-1} c_{v_{j}}}{\sqrt{c_{v_{k}}^{T} Q_{v}^{-1} c_{v_{k}}} \sqrt{c_{v_{j}}^{T} Q_{v}^{-1} c_{v_{j}}}}
$$

where $c_{v_{k}}$ and $c_{v_{j}}$ are zero column vectors except for the elements corresponding to the observations $k$ and $j$, respectively, which equals 1 . If a high correlation coefficient is present for a suspected observation error with other observation errors, one will need to carefully inspect and consider exclusion of these correlated observations one at a time. The Chi-square test (Eq. 4) has to be re-applied after excluding any observation, as well as after removing any of their possible combinations to ensure that the system has no more alternative hypotheses. Usually, code outliers are uncorrelated (El-Mowafy and Kubo 2018); thus, in RTK if cycle slips are successfully detected and repaired, the likelihood of a faulty measurement to mask another would be low.

\section{FDE IN THE POSITION DOMAIN}

The above tests are performed in the observation domain. The FDE can similarly be performed in the position domain using the solution separation method as applied in the ARAIM approach (see for instance Blanch et al. 2014, Jöerger and Pervan 2016; El-Mowafy and Yang 2016). In this method, a position error bound is created for each possible fault mode by computing a position solution unaffected by the fault, computing an error bound around this solution and accounting for the difference between the all-observations position solution and the fault tolerant position. For example, suspecting a fault mode $i$, we compute the position from all observations (denoted as $\hat{x}_{o}$ ) and the position after exclusion of suspected observations, i.e. $\hat{x}_{i}$. In principle, the difference between the two solutions, i.e. the residual positional vector $\Delta \hat{\mathrm{x}}_{i}=\left(\hat{x}_{o}-\hat{x}_{i}\right)$ will be large in the presence of a fault where $\hat{x}_{o}$ will be significantly biased. Normalizing each positional component of this position residual vector $\Delta \hat{\mathrm{x}}_{i}$ by its standard deviation gives a test static that can be assumed in the fault-free unbiased mode has a zero-mean Gaussian distribution. For 2D positioning, the current practice is to test the position components in the East (E) and North (N) directions, and $H_{o}$ can be rejected in favour of the alternative hypothesis, i.e. suspecting a fault in the satellite(s) $i$, when (Blanch et al. 2014):

$$
H_{a_{i_{E}}}: \frac{\left|\Delta \hat{\mathrm{x}}_{i_{E}}\right|}{\sigma_{\Delta \widehat{x}_{i_{E}}}} \geq N_{\frac{\alpha}{4 m}}(0,1) \quad \text { and } \quad H_{a_{i_{N}}}: \frac{\left|\Delta \hat{\mathrm{x}}_{i_{N}}\right|}{\sigma_{\Delta \widehat{x}_{i_{N}}}} \geq N_{\frac{\alpha}{4 m}}(0,1)
$$

where $\Delta \hat{\mathrm{x}}_{i_{E}}$ and $\Delta \hat{\mathrm{x}}_{i_{N}}$ are the vector $\Delta \hat{\mathrm{x}}_{i}$ components in $\mathrm{E}$ and $\mathrm{N}$. For one satellite fault, the errors in $\mathrm{E}$ and $\mathrm{N}$ will be correlated and hence one may choose for the two tailed normal distribution the same significance level $\frac{\alpha}{2}$ for the two components $(E, N)$. However, this will not be the case when more than one satellite fault is considered. Therefore, a conservative general approach is followed here, where the threshold is computed by equally allocating the significance level $\frac{\alpha}{2}$ to $E$ and $N$. The standard deviations (stds) $\sigma_{\Delta \hat{x}_{i}}$ and $\sigma_{\Delta \hat{x}_{i_{N}}}$ are computed from the position VC matrix $Q_{\Delta \hat{x}_{t}}$, which is expressed by applying the error propagation law to KF observation update equation as:

$$
Q_{\Delta \hat{\chi}_{t}}=\left(K_{i} G_{i}-K_{o} G_{o}\right) Q_{\hat{x}_{t, t-1}}\left(K_{i} G_{i}-K_{o} G_{o}\right)^{T}+\left(K_{i} A_{i}-K_{o}\right) Q_{\mathrm{y}}\left(K_{i} A_{i}-K_{o}\right)^{T}
$$

assuming that the two solutions at $t$ have the same predicted (time updated) vector of unknowns $\hat{\chi}_{t, t-1}$, where $K_{o}$ and $K_{i}$ are Kalman gain matrices for the full set of observations and the subset considered in mode $i, G_{o}$ and $G_{i}$ are the corresponding geometry matrices. $Q_{\hat{\chi}}$ denotes the VC matrix of the time update of the unknowns, and $Q_{\mathrm{y}}$ is the VC matrix of the observations, with $m$ is the number of observations. When suspecting $b$ faulty observations, the $A_{i}$ matrix comprises an identity matrix of size $(m-b)$ and $b$ zero column vectors at place of the excluded observations. For instance, if the last observation is excluded, $A_{i}=[I \mid 0]$, where $I$ has a size of $(m-1)$ and 0 is a zero vector. 
The vehicle location is typically expressed in $\mathrm{E}, \mathrm{N}$ coordinates for navigation purpose. In ITS liability- and safetycritical applications, the vehicle position is better expressed along its direction of motion, i.e. by the along-track $(A T)$ and cross-track $(C T)$ horizontal position components (Margaria and Falletti 2014; Imparato et al. 2018). In this case, the position vector and its VC matrix in the (AT, CT, Up) Cartesian frame, define here as $r_{A t-C T-U}$ and $Q_{A t-C T-U}$ respectively, are computed from the position vector and its VC matrix in the $(E, N, U)$ Cartesian frame, i.e. $r_{E-N-U}$ and $Q_{E-N-U}$. Assuming the two coordinate frames have the same scale, the transformation of point coordinates is performed by means of multiplication of clockwise Euler rotation matrices, i.e. through $R_{\zeta, Q, \theta}=R_{\zeta}$ $R_{\varrho} R_{\theta}$, with $\left(R_{\zeta}, R_{\varrho}, R_{\theta}\right)$ are the rotation matrices for the three rotations performed about the axes $(E, N, U)$, respectively, defined by the three attitude angles, the pitch $(\varsigma)$, roll (@) and azimuth $(\theta)$ of the vehicle, such that:

and

$$
r_{A t-C T-U}=R_{\zeta, \varrho, \theta} r_{E-N-U}
$$

$$
Q_{A t-C T-U}=R_{\zeta, Q, \theta} Q_{E-N-U} R_{\zeta, Q, \theta}^{T}
$$

This however requires knowledge of the complete attitude of the vehicle, which can be obtained for example from an Inertial Measurement Unit (or Gyros). If not available, and since the pitch and roll are typically small angles, in particular the roll, an approximation would be to ignore the pitch and roll rotations and use only the azimuth, such as for long straight roads, where the azimuth can be roughly estimated as the direction of the road.

In general, we are not interested in the vertical position in ITS. Hence, limiting our focus to positioning performance in the $A T$ and $C T$ directions; one can reject $H_{o}$ in favour of the alternative hypothesis when:

$$
H_{a_{i A T}}: \frac{\left|\Delta \hat{\mathrm{x}}_{i A T}\right|}{\sigma_{\Delta \widehat{x}_{i}}} \geq N_{\frac{\alpha_{A T}^{\prime \prime}}{2}}(0,1) \quad \text { or } \quad H_{a_{i} C T}: \frac{\left|\Delta \hat{\mathrm{x}}_{i C T}\right|}{\sigma_{\Delta \widehat{x}_{i C T}}} \geq N_{\frac{\alpha_{C T}^{\prime \prime}}{2}}(0,1)
$$

where the $\Delta \hat{\mathrm{x}}_{i}$ and $\Delta \hat{\mathrm{x}}_{i}$ refer to the vector of $\Delta \hat{\mathrm{x}}_{i}$ projected into the $A T$-CT-Up frame. The standard deviations (stds) $\sigma_{\Delta \hat{x}_{i} T}$ and $\sigma_{\Delta \hat{x}_{i} T}$ are computed from the corresponding position VC matrix by error propagation applying the covariance law. Assuming the same total probability of false alarm $(\alpha)$, the significance levels $\alpha_{A T}^{\prime \prime}$ and $\alpha_{C T}^{\prime \prime}$ are expressed as:

$$
\alpha^{\prime \prime}=\frac{\alpha}{2 m} ; \quad \alpha_{A T}^{\prime \prime}=\eta \frac{\alpha}{2 m} \quad \text { and } \quad \alpha_{C T}^{\prime \prime}=(1-\eta) \frac{\alpha}{2 m}
$$

where $\eta$ is a selected weight for $A T$. For instance, assuming equally distributed $\alpha^{\prime \prime}$ for the $A T$ and $C T$ components, we have $\alpha_{A T}^{\prime \prime}=\alpha_{C T}^{\prime \prime}=\frac{1}{2} \alpha^{\prime \prime}$. For testing one alternative hypothesis, the allowable region of the above tests in $2 \mathrm{D}$ is rectangular, different from the Chi-square statistic which has an elliptical allowable region.

In some ITS applications the focus might be in one direction more than the other, for instance, in forward collision warning we have more focus on $A T$ positioning while in signal priority, more focus is given to the $C T$ position component. Hence, in the former example identifying $H_{a_{i}}$ is more important than identifying $H_{a_{i}}$, whereas in the latter example identifying $H_{a_{i} T}$ is of more interest. One here has also to acknowledge that the distribution of visible satellites may lead to the position in one direction be more precise than in the other direction. For example, due to signal obstruction because of the presence of structures and trees on the side of the road, the position component along the $A T$ direction can be more precise than the positioning component in the $C T$ direction. Hence, the presentation of the two binary tests in Eq. (13) might be useful in accepting the solution when one component passes the FDE test while the other does not pass under limited conditions, such as:

- The failed test for one position component is close to the borderline; and a small decrease in $\alpha^{\prime \prime}$ may lead to passing the test.

- The number of satellites is critically low for positioning, i.e. if a satellite in mode $i$ is excluded, the solution may not be available.

This relaxing scenario of partial identification of faults needs further investigation that addresses particular applications and will be addressed in our future research.

\section{FDE in the Urban Environment}

In the urban environment multipath-contaminated signal will distort the correlation function which helps receivers to estimate Time of Arrival of the GNSS signals (Braasch, 1996), where constructive multipath interference leads to an increase in the $\mathrm{C} / \mathrm{N} 0$, while destructive multipath interference leads to a decrease in C/NO. NLOS reception occurs where the direct LOS signal is blocked and the signal is received only via reflection. The measurement error 
is therefore the difference between length of the path taken by the reflected signal and the 'blocked' direct path between the satellite and the receiver. Some techniques were developed to mitigate the effects of multipath, as shown for example in Groves et al., (2013); Mubarak and Dempster (2010); Pirsiavash et al. (2019) and for NLOS (Groves et al., 2012; Hsu et al., 2015).

The above cited models for multipath were mostly developed for static reference station sites, and thus are not suitable for the dynamic environment of transportation applications. In such environment, multipath effects tend to randomize due to the dynamic change in the geometry between reflecting surfaces and the moving receiver, including their distance from the vehicle, and the type and size of reflecting sources. This will cause a stochastic behaviour in addition to a bias. The stochastic part can be included in the observation standard deviations used in their weighting. Typically, in the open environment the observation standard deviation are modelled as a function of the satellite elevation angle. In this study, a model similar to that presented in Tay and Marais (2013) is exploited with the objective of de-weighting the observations that experience large multipath and accordingly reduce their contribution in the solution. The model can be expressed as:

$$
\sigma^{2}=\sigma_{o}^{2} \times \frac{\mathrm{m} \times 10^{-0.1 \times C / N_{o}}}{\sin ^{2}(\theta)}
$$

where $\sigma^{2}$ is the observation variance, $\sigma_{o}^{2}$ is the variance along the zenith direction in the open sky computed using the method described in (El-Mowafy 2014), $m$ is a multiplier for model calibration, which depends on the receiver type and environment. This coefficient is best estimated based on the use of 3D city models (Wang et al. 2012; Hsu et al. 2015), employed to detect which surface of the surrounding buildings could make a specular reflection within a catch circle (taken empirically of $100 \mathrm{~m}$ radius in our study). In our approach, the $m$ values are approximately taken from a lookup table, were the values in the table are computed for a range of three parameters; the satellite elevation and azimuth, and the distance between the receiver and the reflecting surface. One approach for a lesscomplex estimation of $\sigma^{2}$, which would be less optimal, is the use of adaptive Kalman filtering (El-Mowafy and Mohamed 2005).

Since the observation errors in the urban environment do not exactly follow a normal distribution, a paired CDF overbounding technique is applied, see Rife et al. (2006). Its estimation is performed in two steps. Firstly, by determining a unimodal and symmetric distribution $F_{S}$ about its mean, that forms CDF overbounding of the empirical distribution of the data. Next, we determine a Gaussian distribution $N\left(M, \sigma^{2}\right)$ of $F_{S}$, using paired overbounding where a mean $M$ is placed in order to center the CDF. This Gaussian distribution $N\left(M, \sigma^{2}\right)$ is empirically computed based on positioning errors from $\sim 60000$ epochs collected in ten kinematic tests conducted in Australia in different environments, including open sky, semi-urban and urban environments. The thresholds can then be written for $A T$ and $C T$ as:

$$
\begin{aligned}
& H_{a_{i_{A T}}}: \frac{\left|\Delta \hat{x}_{i_{A T}}\right|}{\sigma_{\Delta \hat{x}_{i}}} \geq N_{\frac{\alpha}{4 m}}\left(M_{A T}, \sigma_{A T}^{2}\right) \\
& H_{a_{i_{C T}}}: \frac{\left|\Delta \hat{x}_{i C T}\right|}{\sigma_{\Delta \widehat{x}_{i}} \mid} \geq N_{\frac{\alpha}{4 m}}\left(M_{C T}, \sigma_{C T}^{2}\right)
\end{aligned}
$$

where $\left(M_{A T}, \sigma_{A T}\right)$ and $\left(M_{C T}, \sigma_{C T}\right)$ are the mean (mainly due to multipath) and std for the overbounding distribution in $A T$ and $C T$.

\section{OTHER USEFUL FDE TESTS}

One can argue that the two tests in Eq. (13) may indicate to two alternative scenarios (e.g. a fault in one satellite observation could lead to erroneous $E$ but not $N$, and vice versa). Thus, a practical approach is to combine the two tests into one test and examine one alternative hypothesis, i.e. one faulty satellite observation leads to unacceptable horizontal positioning. Under the assumption that the measurement noise is zero-mean Gaussian, the 2D joint normal distribution function has a 2D sectional view that describes an ellipse as shown in Fig 1. The confidence ellipse thus would represent the sum of squares of two independent normally distributed data. This sum has a Chisquare distribution. Thus, the test can be expressed as:

$$
\frac{\Delta \hat{\mathrm{x}}_{i A T}^{2}}{\sigma_{\Delta x_{i A T}}^{2}}+\frac{\Delta \hat{\mathrm{x}}_{C T}^{2}}{\sigma_{\Delta x_{i C T}}^{2}} \geq \chi_{\frac{\alpha}{m}}^{2}(d f, 0)
$$


and assuming equally distributed $\alpha$ to all $m$ possible fault events (alternative hypotheses).

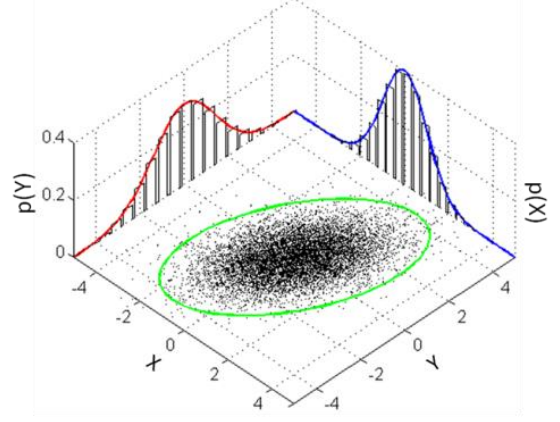

Fig 1. 2D multivariate distribution

In general, neither the $A T$ nor $C T$ errors are the maximum position error that may be experienced along all possible directions. The maximum error is typically aligned with the semi-major axis of a horizontal error ellipse. The maximum error direction can be determined in the Eigen space by the direction of the first Eigen vector. Hence, for the 2D VC matrix of $\Delta \hat{\mathrm{x}}_{i}$, denoted as $\mathrm{Q}_{\Delta \hat{\mathrm{x}}_{i}}$, let $\xi 1_{i}$ be the first Eigenvalue and $\vec{E} 1_{i}$ is the first Eigenvector for this matrix. The semi-major axis of the error ellipse, which represents the max std is $\sigma_{\Delta x_{i_{\max }}}=\sqrt{\xi 1_{i}}$. Hence, we can formulate the test statistic and check along the direction of the maximum error, where the null hypothesis is rejected when:

$$
\frac{\Delta \widehat{\otimes}_{i}^{T \cdot} \vec{E} 1_{i}}{\sigma_{\Delta x_{i \max }}} \geq N \frac{\alpha}{2 m}
$$

assuming equal distribution of $\alpha$ for the maximum and minimum directions. In the 2D space, one would like to consider the joint maximum-minimum case along the semi-major and semi-minor axes of a confidence error ellipse of the $2 \mathrm{D}$ matrix $\mathrm{Q}_{\Delta \hat{\mathrm{x}}_{i}}$; where $\xi 1_{i}$ and $\xi 2_{i}$ denote the the first and second Eigenvalues of $\mathrm{Q}_{\Delta \hat{\Delta}_{i}}$, and $\vec{E} 1_{i}, \vec{E} 2_{i}$ are their corresponding Eigenvectors. By computing $\sigma_{\Delta x_{i_{\max }}}$ and $\sigma_{\Delta x_{i_{\text {min }}}}$, which represent the semi-major (max) and semiminor (min) std, where $\sigma_{\Delta x_{i_{\max }}}=\sqrt{\zeta 1_{i}}$ as mentioned above and $\sigma_{\Delta x_{i_{\min }}}=\sqrt{\xi 2_{i}}$, a fault is suspected when:

$$
\frac{\left(\Delta \widehat{x}_{i}^{T} \cdot \vec{E} 1_{i}\right)^{2}}{\sigma_{\Delta x_{i \max }}^{2}}+\frac{\left(\Delta \hat{x}_{i}^{T} \cdot \vec{E} 2_{i}\right)^{2}}{\sigma_{\Delta x_{i_{\min }}^{2}}^{2}} \geq \chi_{\frac{\alpha}{m}}^{2}(d f, 0)
$$

The elliptical 'allowable' region conforms to the distribution of the position errors. The tests in Eq. 19 and 20 are also useful when the azimuth of the vehicle is not well determined, e.g. during rapid turns, to apply testing in the $A T$ and $C T$ directions.

Figure 2 illustrates the relationships between the error $\Delta x_{i}$ and its projections in the $A T$ and $C T$ directions and along the maximum and minimum directions (defined by the error ellipse), in addition to the test thresholds for the two cases. As the figure shows, in the 2D space, the allowable region for the maximum-minimum (max-min) case for a single mode $i$ is elliptical whereas the allowable region for the AT-CT case, when the test is performed for each component, is rectangular. Hence, for a given significance level, some small threats detected by one model will be acceptable by another. For instance, the point marked by ( $\square)$ will be detected by the $A T$ - $C T$ mode whereas the point marked by $(X)$ will be accepted. The opposite result will happen when applying the max-min model.

A more relaxed direction independent bound for the 2D space can also be established by a circle as shown in Fig 2 for which $\Delta x_{i_{r}}=\sqrt{\Delta \hat{\mathrm{x}}_{i_{A T}}^{2}+\Delta \hat{\mathrm{x}}_{i_{C T}}^{2}}$ and $\sigma_{\Delta x_{i_{r}}}=\sqrt{\sigma_{\Delta x_{i_{A T}}}^{2}+\sigma_{\Delta x_{i_{C T}}}^{2}+2 \sigma_{\Delta x_{i A T}, \Delta x_{i C T}}}$. The 2D position error $\left(\Delta x_{i_{r}}\right)$ can be assumed a random variable, such that an error is suspected when:

$$
\frac{\Delta x_{i_{r}}}{\sigma_{\Delta x_{i_{r}}}} \geq R \frac{\alpha}{m}
$$

with a threshold $R_{\frac{\alpha}{m}}$ which refers to the inverse of the complement of a Rayleigh distribution at a significance level $\frac{\alpha}{m}$. 




Fig 2. Test Thresholds of the $2 \mathrm{D}$ projections $(A T \& C T)$ and (max \&min) of the error ellipse

In summary, as mentioned earlier, positioning can be expressed in ITS according to the application at hand. Table 1 recaps the different FDE parametrization models discussed in this paper and their usage. For instance, the position of a vehicle can be expressed on navigation maps using the Easting and Northing coordinates. In the liability-critical applications, such as tolling and signal priority, the cross-track position is needed for lane identification. In safetycritical applications, such as collision avoidance, the position is expressed in the along-track and cross-track directions. The joint statistic has an advantage in applying only one test, and the joint max/min FDE is designed when the direction of the vehicle is changing rapidly during sharp turns. Finally, in urban environments, FDE is formulated taken into consideration multipath effects. The switch between these testing models, can be easily performed by the computer of the vehicle according to the vehicle status and application.

Table 1. Summary of detection tests in the position domain

\begin{tabular}{|c|c|c|c|c|}
\hline Type & ITS Usage & Statistic & Threshold & $\begin{array}{l}\text { Detection } \\
\text { region }\end{array}$ \\
\hline$E, N$ & Mapping & $\frac{\left|\Delta \hat{x}_{i_{E}}\right|}{\sigma_{\Delta \widehat{x}_{i_{E}}}} ; \frac{\left|\Delta \widehat{x}_{i_{i}}\right|}{\sigma_{\Delta \widehat{x}_{i_{N}}}}$ & $N_{\frac{\alpha}{4 m}}(0,1)$ & Rectangular \\
\hline$A T, C T$ & $\begin{array}{l}\text { liability and safety } \\
\text { applications }\end{array}$ & $\frac{\left|\Delta \hat{x}_{i A T}\right|}{\sigma_{\Delta \hat{x}_{i A T}}} ; \frac{\left|\Delta \hat{x}_{i C T}\right|}{\sigma_{\Delta \hat{x}_{i C T}}}$ & $N_{\frac{\alpha}{4 m}}(0,1)$ & Rectangular \\
\hline $\begin{array}{l}\text { Joint } A T, \\
C T\end{array}$ & $\begin{array}{l}\text { One test for liability and } \\
\text { safety }\end{array}$ & $\frac{\Delta \hat{\mathrm{x}}_{A T T}^{2}}{\sigma_{\Delta x_{i} T}^{2}}+\frac{\Delta \hat{\mathrm{x}}_{C T}^{2}}{\sigma_{\Delta x_{i} C T}^{2}}$ & $\chi_{\frac{\alpha}{m}}^{2}(d f, 0)$ & elliptical \\
\hline $\begin{array}{l}\text { Joint } \\
\max , \min \end{array}$ & $\begin{array}{l}\text { Direction is changing } \\
\text { rapidly }\end{array}$ & $\frac{\left(\Delta \hat{\mathrm{x}}_{i}^{T} \cdot \vec{E} 1_{i}\right)^{2}}{\sigma_{\Delta x_{i_{\max }}^{2}}^{2}}+\frac{\left(\Delta \hat{\mathrm{x}}_{i}^{T} \cdot \vec{E} 2_{i}\right)^{2}}{\sigma_{\Delta x_{\text {min }}}^{2}}$ & $\chi_{\frac{\alpha}{m}}^{2}(d f, 0)$ & Elliptical \\
\hline$A T, C T$ & in urban environment & $\frac{\left|\Delta \hat{x}_{i A T}\right|}{\sigma_{\Delta \hat{x}_{i A T}}} ; \frac{\left|\Delta \hat{x}_{i C T}\right|}{\sigma_{\Delta \hat{x}_{i C T}}}$ & $N_{\frac{\alpha}{4 m}}\left(M_{A T}, \sigma_{A T}^{2}\right) ; N_{\frac{\alpha}{4 m}}\left(M_{C T}, \sigma_{C T}^{2}\right)$ & Rectangular \\
\hline
\end{tabular}

If a fault is detected, we consider exclusion of the observations in the fault mode $i$. To verify this exclusion and to avoid wrong exclusion an additional test is performed. A test statistic is formed as the difference between the solution using the observation subset excluding the suspected faulty observations, i.e. $\hat{\mathrm{x}}_{i}$, and the solution using other subsets that exclude one of the remaining observations, denoted as $\hat{\mathrm{x}}_{j}$. For example, in the $A T$-CT case:

$$
\frac{\left|\left(\hat{\mathrm{x}}_{i}-\hat{x}_{j}\right)_{A T}\right|}{\sigma_{\Delta x_{i-j}}{ }_{A T}}>N_{\frac{\alpha_{A T}^{\prime \prime}}{2}}(0,1) \text { and } H_{a_{i} C T}: \frac{\left|\left(\hat{x}_{i}-\hat{x}_{j}\right)_{C T}\right|}{\sigma_{\Delta x_{i-j}}}>N_{\frac{\alpha_{C T}^{\prime \prime}}{2}}(0,1)
$$

The stds $\sigma_{\Delta x_{i-j}}$ and $\sigma_{\Delta x_{i-j}}$ are computed from the covariance matrix, such that:

$$
Q_{\Delta \hat{\mathrm{\chi}}_{i-j}}=\left(K_{i} G_{i}-K_{j} G_{j}\right) Q_{\hat{\mathrm{\chi}}_{t, t-1}}\left(K_{i} G_{i}-K_{j} G_{j}\right)^{T}+\left(K_{i}-K_{j}\right) Q_{\mathrm{y}}\left(K_{i}-K_{j}\right)^{T}
$$


where $K_{j}$ and $G_{j}$ are the Kalman gain and geometry matrices for the subset excluding measurement $j$. If the test passes, one can conclude that the two solutions are inconsistent, which supports exclusion of the observations in fault mode $i$. However, if the test fails, one has to consider two options; either there are still wrong observations remaining, or the excluded observations might not be actually the faulty ones. A study of the correlation between the observation errors and the possibility of excluding them all together may then be considered if a solution is still possible; if not, a solution is declared invalid.

\section{RELATIONSHIP BETWEEN THE SOLUTION SEPARATION AND CHI-SQUARE TESTS}

As shown from Figure 2, for each fault mode, the Solution Separation (SS) allowable region for FD in the position domain when considering the $2 \mathrm{D}$ components $(E, N)$ or $(A T, C T)$ is rectangular and elliptical for the Chi-square test. For the multi-dimension case, the SS no-detection and exclusion regions are polytopic and prismatic when considering the position components $(E, N, U)$, whereas for Chi-square they are hyperspherical and cylindrical, respectively (Jöerger et al. 2014). As such, some errors detected by one method could be missed by the other and vice versa. (Imparato 2014) agreed, and showed that the two methods differ in case of multi-satellite faults occurring at the same time. The two statistics; the Chi-square and the SS, are projected onto two orthogonal spaces. Thus, this would create special cases. For instance, in the rare event that the error is completely mapped onto the space of Chisquare, it will not be mapped onto the space of the SS test statistic, which means that the error does not have an effect on the solution in the position domain at the user location, and as such it will not be detected by the SS test. In this case, the Chi-square will rightfully reject the null hypothesis, whereas the SS will accept it.

Some differences may also be experienced because the SS test statistic detects only the outliers that eventually affect the position solution. This means that there could be outliers present that have a counter effect of each other on the final position, leading to a position error at specific user locations, which will not be detected by the SS method but would be detected by the Chi-square test. In integrity monitoring, Blanch et al. (2015) recommended that if the Chisquare detection test fails but the SS detection test passes for all position components; the fault is considered outside the assumed threat model in the SS test, and integrity of the system at this epoch can be assumed unavailable.

\section{THE FINAL INTEGRITY TEST 'FIT'}

A new test called the Final Integrity Test (FIT) is presented. The test quantifies the impact of the error vector $\widehat{\nabla}_{t}$, computed from the observations passing the FDE, on the position solution and checks whether this positioning error is less than an allowable error by the ITS application considered. This positioning error vector, denoted as $\delta \hat{x}_{t}$ (also known in the reliability theory as the external reliability), can be expressed in the recursive Kalman filter process as (Teunissen 2006):

$$
\delta \hat{x}_{t}=K_{t} C_{t} \widehat{\nabla}_{t}
$$

where $K_{t}$ is the gain matrix at time $t$, and $\widehat{\nabla}_{t}=\left(C_{t}^{T} Q_{v_{t}}^{-1} C_{t}\right)^{-1} C_{t}^{T} Q_{v_{t}}^{-1} \hat{v}_{t}$. $C_{t}$ is a square matrix of the size of the number of accepted observations (hence becomes an identity matrix); and thus $\widehat{\nabla}_{t}$ includes the effect of the predicted residuals of all accepted observations.

The FIT test introduced here checks that the $A T$ and $C T$ components of $\delta \hat{x}_{t}$, i.e. $\delta \hat{x}_{t}$ and $\delta \hat{x}_{t_{C T}}$ satisfy the conditions:

$$
\left|\delta \hat{x}_{t_{A T}}\right|<\mu_{A T} \text { and }\left|\delta \hat{x}_{t_{C T}}\right|<\mu_{C T}
$$

to accept the position, where $\mu_{A T}$ and $\mu_{C T}$ are the maximum allowable errors for $A T$ and $C T$, respectively, selected based on the application at hand. An overall horizontal check can also be expressed as:

where

$$
\delta \hat{x}_{t_{H}}<\mu_{H}
$$

$$
\delta \hat{x}_{t_{H}}=\sqrt{\left(\delta \hat{x}_{t_{A T}}\right)^{2+}\left(\delta \hat{x}_{t_{C T}}\right)^{2}} \text { and } \mu_{H}=\sqrt{\mu_{A T}^{2}+\mu_{C T}^{2}}
$$

For example, in ITS applications that require lane identification, the allowable $C T$ error $\left(\mu_{C T}\right)$ can be selected as $1 \mathrm{~m}$, whereas for more precise applications, such as forward collision alerts, the allowable $A T$ error $\left(\mu_{A T}\right)$ can be set as $0.2-0.5 \mathrm{~m}$. If the test passes and the precision results are satisfactory, the positioning solution is accepted. If the test fails, this position cannot be validated and an alternative positioning system should be tried. 


\section{TESTING}

To demonstrate the performance of the various FDE models presented in a practical environment of ITS applications, a kinematic test was conducted in Australia using a vehicle fitted with a multi-frequency GNSS receiver. The test was conducted for approximately $3.5 \mathrm{hrs}$ with $1 \mathrm{~Hz}$ data sampling rate and the trajectory comprised various possible environments that may be experienced in ITS, including suburban with good satellite visibility, and for some periods near to tree canopies and an urban environment where signals were subject to obstructions (but still mostly have enough number of satellites for positioning in this test). Only GPS data was used where 2D positioning was considered, which is of primary interest in ITS. Positioning was performed in a network real-time kinematic (NRTK) method, where the collected satellite observations were post-processed with the network data for testing various FDE methods. The test trajectory and sky plot of the observed satellites during testing are illustrated in the Figures 3 and 4, respectively. The gaps seen at the bottom of figure 3 refer to regions with unavailable positioning due to observing a limited number of satellites.

To indicate which areas that may have faults in the NRTK observations, Figure 5 shows the time series of the position errors in $\mathrm{E}$ and $\mathrm{N}$, computed as the difference between the NRTK solution, without FDE, and an integrated GPS/Inertial Measurement unit (GPS/IMU) loosely coupled post-processing solution. The GPS positioning in this GPS/IMU solution was obtained from post processing the vehicle stored GPS observations with FDE in a relative mode with data from a base station that was located a few kilometres away, and only ambiguity-fixed solutions were used that delivers a few $\mathrm{cm}$ accuracy. The GPS/IMU provides a good solution, particularly in the urban environment when satellite visibility is low. The shaded areas in the figure indicate the urban environment which is characterized by the presence of buildings of two stories or above on both sides of the road, leading to a low sky visibility, presence of multipath and NLOS, which all led to a significant degradation of positioning availability and accuracy. The remaining parts in the plot indicate suburban areas that have good satellite visibility and geometry.

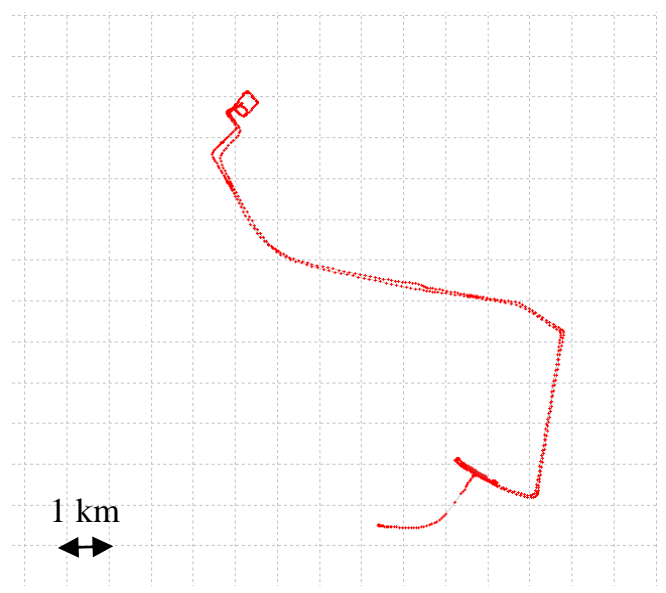

Fig 3. Test trajectory

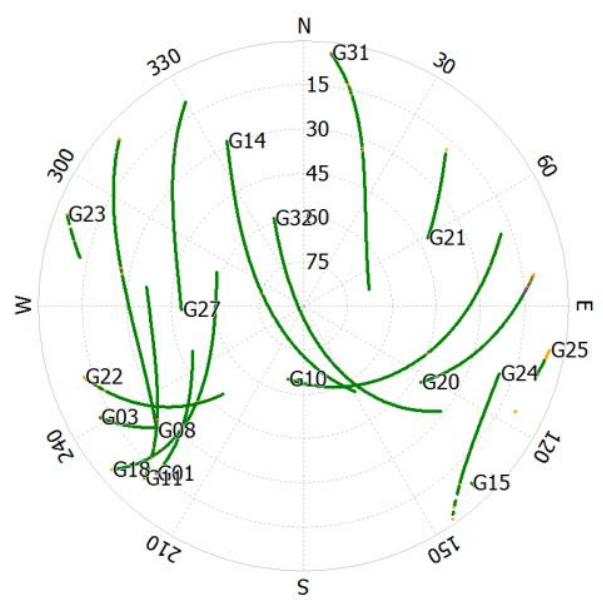

Fig 4. Sky plot of the test GPS satellites

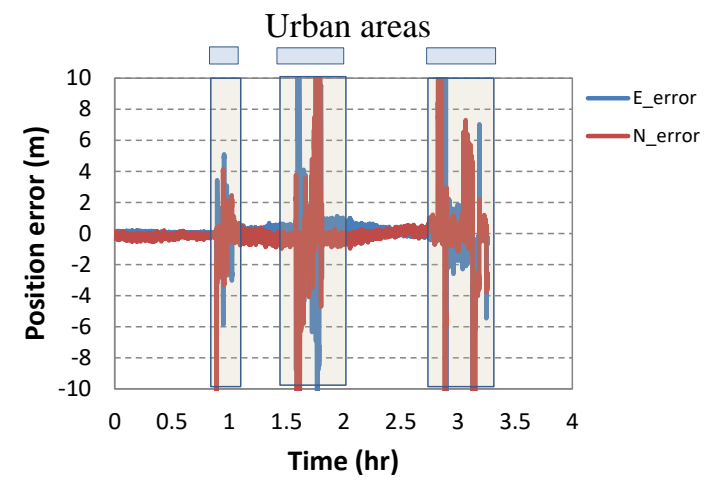

Fig 5. Position errors in $\mathrm{E} \& \mathrm{~N}$, The shaded areas indicate urban environment with low sky visibility, and presence of multipath and NLOS 
Identification was performed for the case of single and multiple faults (up to the degrees of freedom as discussed earlier). To save the computational time, once a fault is identified and verified (using Eq. 9 or 22), the Chi-square detection test is applied for the remaining observations, and if passes, the identification search stops. The performance of various FDE test parametrizations in the different ITS environments, which vary from working under good satellite visibility and geometry conditions to poor conditions, is demonstrated through the Figures 6 to 10. The urban areas are marked above the figures. Firstly, Figure 6 shows the time series of the Chi-square test results, illustrating the statistic and the test threshold, which vary according to the number of the observed satellites and accordingly the degrees of freedom. One can see that due to low number of observations in the urban part of the trajectory, and with multipath and NLOS, the test did not pass for the majority of instances in this environment indicating the presence of faults, whereas there were no faults detected in the suburban environment where good satellite geometry and number was available. For the solution separation (SS) method, and for demonstration purpose, only one representative example is shown where one satellite (PRN10), was excluded when computing $\hat{x}_{i}$. Figures 7 to 10 illustrate the time series of the corresponding statistics and thresholds for FDE in the position domain when parameterizing the test for $(E, N),(A T, C T)$, (maximum, minimum) and finally the circular FDE, respectively (note the open sky and urban areas), where the $K$ symbol in the figures refers to the threshold. As shown in the figures, and similar to the Chi-square test, multiple faults were detected in the urban environment. One can also note that the detection performance of various FDE tests is very close. The subtle differences among them are attributed to the small difference in the shape of their detection regions as discussed earlier when the statistic values were close to the threshold values.

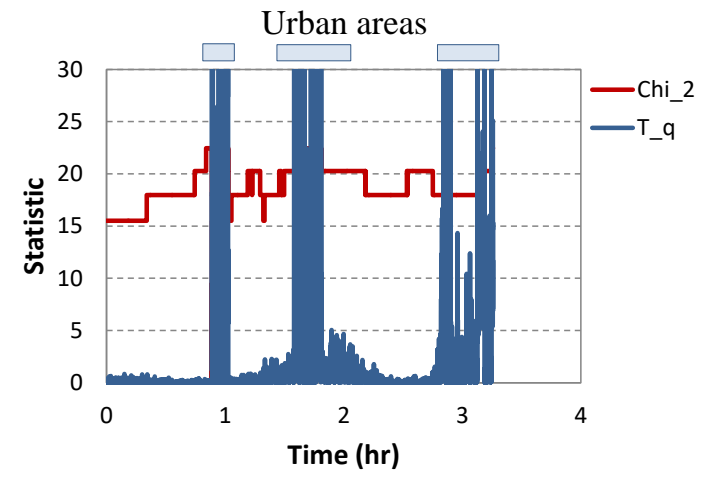

Fig 6. Chi-square test results, $T_{q}=\left(\hat{\nabla}^{T} Q_{\widehat{\nabla}}^{-1} \hat{\nabla}\right)$ (excluding PRN 10)

Table 2 summarises the detection percentage, i.e. the percentage of the number of epochs when faults were detected with respect to the total number of epochs in the test. Among the detected faults, the percentage of epochs where multiple and single detected faulty observations are given in the second and third rows of the table, respectively. For example, in the Chi-square test, among the detections, one can see that $79 \%$ were with multiple faults and $29 \%$ were with a single fault. The number of multiple identified faults, which were primarily code outliers, was larger than the number of single faults, mainly because these faults took place in the urban environment, where multiple signals experience large multipath effects. The table shows that Chi-square results were the closest to the maxim/minimum model results, and the direction-independent circular test gave less detection due to having a larger allowable region. These results agree with the results of the proposed FIT test applied after removal of satellite 10, shown in Figure 11. The figure depicts the time series of the impact of the measurement residuals on the position (i.e. $\delta \hat{x}_{t_{H}}$ ) versus a threshold value $\mu_{\mathrm{H}}$, selected as $1 \mathrm{~m}$ in this demonstration using NRTK, which is suitable for lane identification applications. The small values of $\delta \hat{x}_{t_{H}}$ refer to the periods when the vehicle was in an open sky environment, whereas the large values when $\delta \hat{x}_{t_{H}}$ exceeded $\mu_{\mathrm{H}}$ refer to most of the periods when the vehicle was in an urban environment or near to tree canopies. 
Table 2. Detection percentages (\%) (i.e. percentage of the number of epochs where faults were detected with respect to the total number of epochs) in various methods, and percentage of epochs with single \& multiple faults with respect to the total number of epochs of detected faults

\begin{tabular}{lccccc}
\hline & Chi-square & $\mathrm{SS}(E, N)$ & $\mathrm{SS}(A T, C T)$ & $\mathrm{SS}(\max , \min )$ & $\mathrm{SS}(R)$ \\
\hline Detection \% & 14.83 & 14.32 & 15.67 & 15.64 & 72 \\
\hline $\begin{array}{l}\text { \% of epochs of multiple faults to the } \\
\text { total of epochs with detected faults }\end{array}$ & 71 & 70 & 73 & 28 & 71 \\
\hline $\begin{array}{l}\text { \% of remaining epochs, that of single } \\
\text { faults to the total of epochs with } \\
\text { detected faults }\end{array}$ & 29 & 30 & 27 & 28 & 29 \\
\hline
\end{tabular}

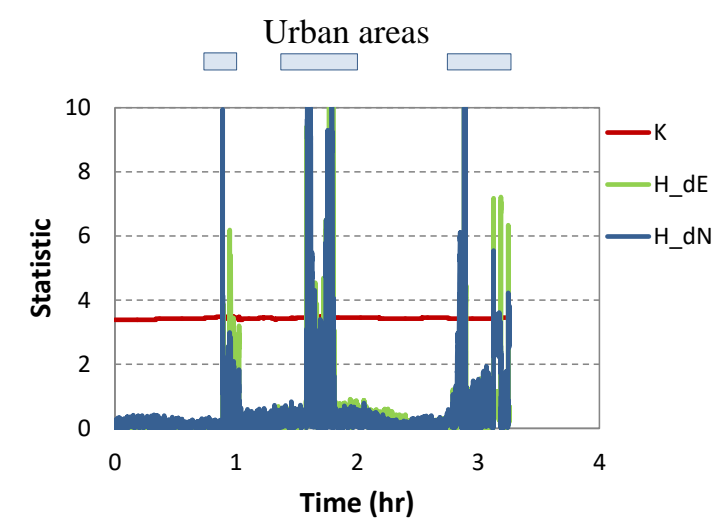

Fig 7. $E, N$ test results (excluding PRN 10) statistic: $\mathrm{H} \_\mathrm{dE}=\frac{\Delta \widehat{\mathrm{x}}_{i_{E}}}{\sigma_{\Delta \widehat{x}_{i_{E}}}}$ and H_dN= $\frac{\Delta \hat{\mathrm{x}}_{i_{N}}}{\sigma_{\Delta \widehat{x}_{i_{N}}}}$

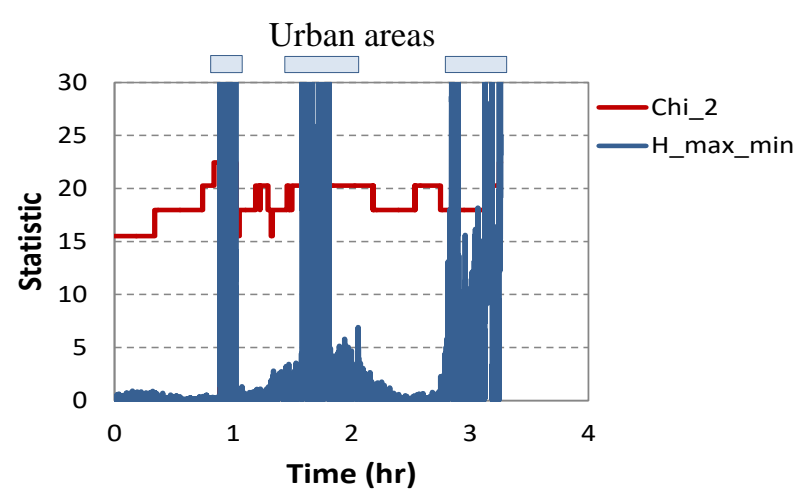

Fig 9. Max, min test results (excluding PRN 10) statistic: $H \_m a x \_m i n=\frac{\left(\Delta \hat{\mathrm{x}}_{i}^{T} \cdot \vec{E} 1_{i}\right)^{2}}{\sigma_{\Delta x_{i \max }}^{2}}+\frac{\left(\Delta \hat{\mathrm{x}}_{i}^{T} \cdot \vec{E} 2_{i}\right)^{2}}{\sigma_{\Delta x_{i_{\min }}}^{2}}$

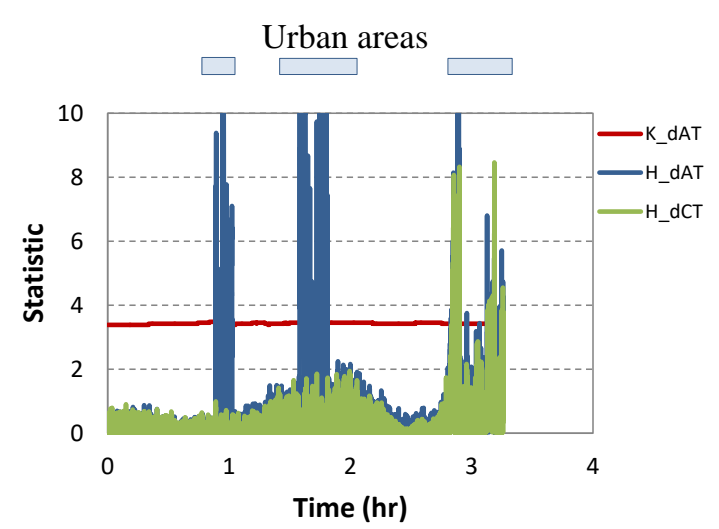

Fig 8. $A T, C T$ test results (excluding PRN 10) statistic: H_dAT $=\frac{\Delta \hat{\mathrm{x}}_{i_{A T}}}{\sigma_{\Delta \hat{x}_{i A T}}}$ and H_dCT $=\frac{\Delta \hat{\mathrm{x}}_{i C T}}{\sigma_{\Delta \widehat{x}_{i C T}}}$



Fig 10. Circular results (excluding PRN 10) statistic: H_R $=\frac{\Delta x_{i_{r}}}{\sigma_{\Delta x_{i_{r}}}}$

Urban areas

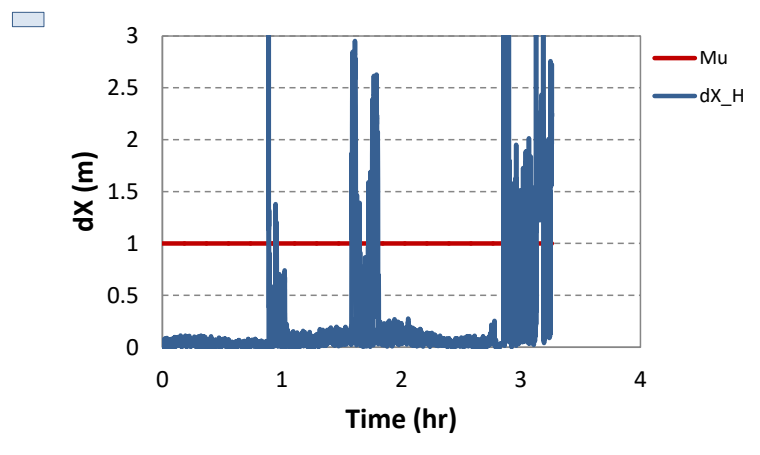

Fig 11. Results of the 2D FIT test (excluding PRN 10) 
We firstly experimentally verified that all methods can detect large errors by inserting 420 artificial errors of 100 $\mathrm{m}$, scattered over 30 minutes, in the code observations in the data of the open sky environment section (not shown in the figures). All these large synthetic errors were detected by all methods. In addition, and without using synthetic errors, detection of faults in the actual observations is verified by comparing the positions computed at the epochs where faults were detected (without excluding the detected faults) with positions obtained from the independent GPS/IMU integration as mentioned above, where these positions came mainly from IMU. A large discrepancy between the two solutions, would show that the GNSS solution is indeed contaminated by faults. Moreover, in the urban environment, we found that the epochs of detected faults agree with epochs where large multipath was detected by the $3 \mathrm{D}$ city models.

For smaller errors, the differences between these fault detection tests are subtle and are due to the changes in their detection region. These differences for small errors do not really depend on having a single or multiple faults but rather on the 'combined' effect of the fault(s) as projected in the position domain, where the test statistics are presented. The proposed FDE for urban environment (Eq. 15-17) was next experimented by re-processing the data and applying the urban test only in the urban areas. The method can be activated in practice by dividing the road into segments and identifying the urban areas based on features from the 3D maps. From results depicted in Figure 12 , when applying the tests for the urban marked areas, one can see the test statistics reduced in size compared with those shown in figure 8 when the observations that experience multipath are deweighted. This also resulted in a less number of observations being rejected as their manifestation did not, for many epochs, present a threat that can significantly bias the solution and as the test threshold adapts to the position error distribution under multipath conditions (see the change in the threshold in Figure 12). However, due to a limited number of observations in the urban environment, which leads to weak modelling that can produce incorrect ambiguity fixing, several epochs did not pass the test. Overall, the number of rejected epochs using this method was $27 \%$ less than when using Eq 13. In the urban areas, the epochs of detected faults agree with epochs that have large multipath as detected by the 3D city models.

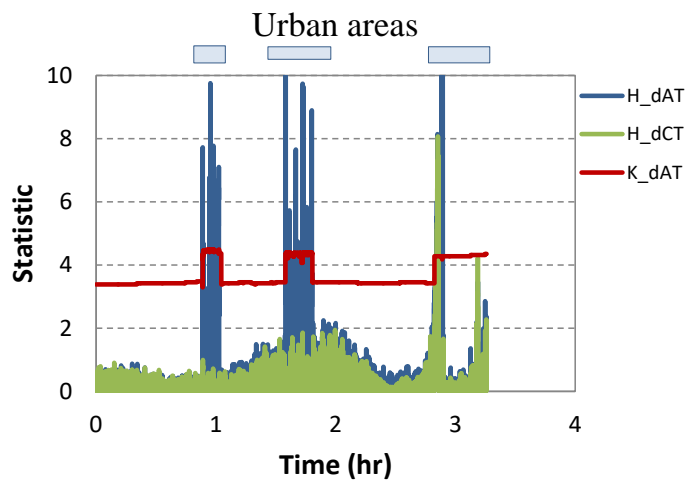

Fig 12. FDE results using the urban model (applied only in the marked urban areas)

$$
\text { statistic } \mathrm{H} \_\mathrm{dAT}=\frac{\Delta \hat{\mathrm{x}}_{i_{A T}}}{\sigma_{\Delta \widehat{x}_{i A T}}} \text { and } \mathrm{H} \_\mathrm{dCT}=\frac{\Delta \hat{x}_{i C T}}{\sigma_{\Delta \hat{x}_{i C T}}}
$$

One should note that the coefficients used in the FDE test in the urban environment are empirically estimated and may need modifications in other areas/environments. Due to the locality of the multipath phenomenon, this issue will always be challenging. In addition, identifying the multipath-contaminated signal from the 3D city models by studying different possible surfaces is still a time consuming process that could hinder its application in real time. Therefore our future research will focus on FDE in the urban environment. In particular, research the reduction of the processing time; better identification of the observations that may experience multipath with the help of 3D city models, eye-fish cameras, or with the help of LiDAR when being integrated with GNSS; possible improvement in deweighting models of multipath effects, and improvements in the paired CDF overbounding technique that is applied to estimate the test threshold.

\section{CONCLUSION}

Positioning is a critical component of ITS, where fault detection is a main task needed to ensure positioning reliability. In this work, two approaches in fault detection with different possible models are discussed. In the observation domain the Chi-square test is modelled in terms of the predicted observation residuals and the corresponding observation errors. In the position domain, different FDE parameterizations are presented based on the solution separation concept. FDE is applied for the Easting and Northing horizontal components for navigation. 
Since ITS liability and safety critical applications are more interested in the direction of motion of the vehicle, FDE models for both the along-track (AT) and cross-track (CT) horizontal position components are presented and to their combined form introducing a single test. Additional forms are presented for the maximum position error, and for the joint maximum-minimum case, needed when the attitude of the vehicle changes rapidly. Finally, a relaxed direction-independent circular bound model is presented.

The performance of the proposed models was demonstrated in a kinematic test that undergoes variable environments that can be experienced in practice in ITS, including open sky, urban and near to tree canopies. All methods detect large errors, but for smaller errors close to their thresholds the differences in results between these tests were subtle, which were due to the change of the shapes of their detection regions. The method can be selected by the on-board computer based on the vehicle status and application as described in Table 1. In general, the results deteriorated with the reduction of the number of the used satellites in positioning. These results agree with the proposed FIT test. In the urban environment and due to multipath and having a low number of observations, even without the presence of faults in the observations the large observation residuals (in Chi-square) and the corresponding large positioning errors in the solution separation method may lead to failing the detection tests. Therefore, a new FDE in the urban environment is presented, where first the observations that may experience multipath are identified with the help of 3D city models and are deweighted. Next a paired CDF overbounding technique was applied to estimate the fault detection test threshold. This method showed improvement compared with the other methods but its coefficients are empirical and it still suffers from the long time needed to quantify the impact of multipath from different surfaces. Therefore, our future research will focus on FDE in the urban environment.

\section{ACKNOWLEDGEMENT}

This research is supported by the Australian Research Council grant number DP170103341. Some of the data used were collected during an SBAS-testbed experiment, grant number, PD8703 funded by FrontierSI, and Geoscience Australia. Norman Cheoung and Joon Wayn Cheong are acknowledged for their help in data collection.

\section{AUTHOR CONTRIBUTION AND DATA AVAILABILITY}

The author has developed the theory, performed the computations and data analysis, and wrote the manuscript.

The data that support the findings of this study are available from the corresponding author, upon reasonable request.

\section{REFERENCES}

Aggarwal, P, Syed Z, Noureldin A, El-Sheimy N (2010) MEMS-Based Integrated Navigation. Artech House Publisher, Norwood, UK.

Baarda WA (1968) Testing Procedure for Use in Geodetic Networks Netherlands Geodetic Commission, Publications on Geodesy, New Series, 2(5).

Blanch, J, Walter T, Enge P (2014) Optimal Positioning for Advanced RAIM, Navigation, 60(4): 279-289.

Blanch J, Walter T, Enge P, Lee Y, Pervan B, Rippl M, Spletter A, Kropp V (2015) Baseline advanced RAIM user algorithm and possible improvements, IEEE Transactions on Aerospace and Electronic Systems. 51(1):713-732.

Braasch, M. S. (1996) Multipath effects," in Global Positioning System: Theory and Applications, vol. 1, B. W. Parkinson and J. J. Spilker, Jr., Eds. Washington, DC, USA: AIAA, ch. 14, pp. 547-568.

Brow R.G. (1992) A Baseline GPS RAIM Scheme and a Note on the Equivalence of Three RAIM Methods. Navigation. 39(3), 301-316.

De Bakker PF, Van der Marel H, Teunissen PJG (2009) The Minimal Detectable Bias for GNSS Observations with a Single Receiver Setup and a Geometry-Free Model. Proceedings of ENC-GNSS 2009, Naples, Italy, 3-6 May 2009.

El-Mowafy A, Mohamed A (2005) Attitude Determination from GNSS Using Adaptive Kalman Filtering, Journal of Navigation, 58(1): 135-148.

El-Mowafy A (2014) GNSS Multi-frequency Receiver Single-Satellite Measurement Validation Method, GPS Solutions, 18(4): 553-561.

El-Mowafy A (2015) Diagnostic Tools Using a Multi-Constellation Single-Receiver Single-Satellite Data Validation Method, J. of Navigation, 68(1): 196-214.

El-Mowafy A, Yang C (2016) Limited Sensitivity Analysis of ARAIM Availability for LPV-200 over Australia using real data. Advances in Space Research, 57(2): 659-670.

El-Mowafy A, Kubo N (2017) Integrity Monitoring of Vehicle Positioning in Urban Environment Using RTKGNSS, IMU and Speedometer. Measurement, Science and Technology, 28(5) 055102, 1-12.

El-Mowafy A (2017) Advanced Receiver Autonomous Integrity Monitoring Using Triple Frequency Data with a Focus on Treatment of Biases. Advances in Space Research, 59(8): 2148-2157. 
El-Mowafy A, Kubo N (2018) A new Approach for Positioning Integrity Monitoring of Intelligent Transport Systems Using Integrated RTK-GNSS, IMU and Vehicle Odometer, IET Intelligent Transport Systems, 12(8), 901-908.

El-Mowafy A, Imparato D (2018) Positioning Integrity, Availability and Precision for Journey Planning and Navigation using GNSS Integrated with Low-Cost Sensors. Proceedings. ION GNSS+ 2018, September 24-28, 2018, Miami, Fl.

Groves PD, Jiang Z, Wang L, Ziebart (2012) M. Intelligent urban positioning using multi-constellation GNSS with 3D mapping and NLOS signal detection. In Proceedings of the ION GNSS 2012, Nashville, Tennessee, 458 472.

Hsu L, Gu Y, Kamijo S (2015) NLOS Correction/Exclusion for GNSS Measurement Using RAIM and City Building Models, Sensors, 15:17329-17349.

Imparato D (2014) Detecting multi-dimensional threats: A comparison of solution separation test and uniformly most powerful invariant test. Proceedings of the European Navigation Conference (ENC)-GNSS. 7-14 April 2014. pp.1-13

Imparato D, El-Mowafy A, Kubo N (2018) Integrity Assessment of Vehicle Positioning for Journey Planning in Urban Environment using RTK and 3D City Models, J. of Intelligent Transport Systems: Technology, Planning, and Operations.

Imparato D, El-Mowafy A, Rizos C (2018) Positioning Integrity Monitoring: from Aviation to Land Applications. Multifunctional Operation and Application of GPS. InTech Publisher, UK, Chapter 2, 23-43.

Intergovernmental Committee on Surveying and Mapping (ICSM) 2007, Standards and Practices for Control Surveys - Special Publication 1 (version 1.7) Canberra Australia.

Jöerger M, Chan FC, Pervan B (2014) Solution separation versus residual-based RAIM. Navigation, 61(4): 273291.

Jöerger M, Pervan B (2016) Fault Detection and Exclusion Using Solution Separation and Chi-Squared ARAIM, IEEE Transactions on Aerospace and Electronic Systems, 52(2): 726-741.

Knight N, Wang J, Rizos C (2010) Generalised measures of reliability for multiple outliers, J of Geodesy, 84(10):625-635.

Krarup T, Kubik K, Juhl J (1980) Götterdämmerung Over Least Squares. In: Proceedings of International Society for Photogrammetry 14th Congress. Hamburg, pp 370-378.

Kuusniemi, H. (2005) User-Level Reliability and Quality Monitoring in Satellite-Based Personal Navigation. PhD dissertation, The University of Calgary, pp209.

Leick A (2004) GPS Satellite Surveying. JohnWiley and Sons, Inc., Hoboken, New Jersey, 3rd edition.

Margaria D, Falletti E (2014) A novel local integrity concept for GNSS receivers in urban vehicular contexts. Proceedings of the IEEE/ION PLANS 2014, Monterey, CA, USA, 5-8 May, 413-425.

Mubarak OM, Dempster AG (2010) Analysis of early late phase in single-and dual-frequency gps receivers for multipath detection. GPS Solutions, 14(4): 381-388.

Parkinson B. W. and P. Axelrad (1987) A Basis for the Development of Operational Algorithms for Simplified GPS Integrity Checking, Proc. of the First Technical Meeting of the Satellite Division of the Institute of Navigation, Colorado Springs, Colorado USA, 269-276.

Pirsiavash A, Broumandan A, Lachapelle G, O'Keefe K (2019) Detection and De-weighting of Multipath-affected Measurements in a GPS/Galileo Combined Solution, In Proceedings of the European Navigation Conference 2019, 9-12 April, Warsaw, Poland, pp. 1-12, 2019.

Powe M, Owen J (1997) A flexible RAIM algorithm. Proc. ION GPS 1997, Kansas City, Sept 1997, 439-449.

Rife J, Pullen s, Enge P, Pervan B (2006) Paired Overbounding for Nonideal LAAS and WAAS Error Distributions, IEEE Trans. On Aerospace and Electronic Systems,42(4), 1386-1395.

Tay S, Marais J. (2013) Weighting models for GPS Pseudorange observations for land transportation in urban canyons. 6th European Workshop on GNSS Signals and Signal Processing, Dec 2013, Germany. 4p. hal00942180.

Santa J, Ubeda B, Toledo R, Skarmeta AFG (2006) Monitoring the Position Integrity in Road Transport Localization Based Services. IEEE Vehicular Technology Conference, Montreal, Que., 2006, 1-5.

Sturza M (1988) Navigation system integrity monitoring using redundant measurements. Navigation, 35(4): 483501.

Teunissen PJG, Kleusberg A (1998) GPS for Geodesy, 2nd ed., Springer, NY.

Teunissen PJG (2000) Testing theory: an introduction. Delft University Press Series on Mathematical Geodesy and Positioning.

Teunissen PJG (2006) Testing theory: an introduction. 2nd ed., Delft VSSD, The Netherlands.

Walter T, Enge P (1995) Weighted RAIM for precision approach. Proceedings of the ION GPS-95, Palm Springs, 12-15 September, 1995-2004.

Walter T, Blanch J, Choi J, Reid T, Enge P (2013) Incorporating GLONASS into Aviation RAIM Receivers, Proceedings of the 2013 International Technical Meeting of The ION, 27-29 January, 239 - 249. 
Wang L, Groves PD, Ziebart MK (2012) Multi-constellation GNSS performance evaluation for urban canyons using large virtual reality city models, Journal of Navigation, 65(3): 459-476.

Wieser. A. (2001) Robust and Fuzzy Techniques for Parameter Estimation and Quality Assessment in GPS. PhD thesis, Graz University of Technology, Graz, Austria, 2001.

Zhu N, Betaille D, Marais J, Berbineau M (2018) GNSS Position Integrity in Urban Environments: A Review of Literature. IEEE Transactions of Intelligent transport systems, 19(9): 2762-2778. 